December 17, 2005. Revised April 13, 2006.

\title{
A quenched CLT for super-Brownian motion with random immigration
}

\author{
Wenming Hong 1 and Ofer Zeitoun:2
}

\begin{abstract}
A quenched central limit theorem is derived for the super-Brownian motion with super-Brownian immigration, in dimension $d \geq 4$. At the critical dimension $d=4$, the quenched and annealed fluctuations are of the same order but are not equal.
\end{abstract}

Key words: Super-Brownian motion, quenched central limit theorem, random immigration .

Mathematics Subject Classification (2000): Primary 60J80; Secondary 60F05.

\section{Introduction and statement of results}

Super-Brownian motion with super-Brownian immigration (SBMSBI, for short), is a superprocess in random environment, where the environment is determined by an immigration process which is controlled by the trajectory of another super-Brownian motion. Many interesting limit properties for SBMSBI were described under the annealed probability ([H02, [H03, HL99] and [Zh05]). In this paper, we study the central limit theorem (CLT) under the quenched probability, that is, conditioned upon a realization of the immigration process, for $d \geq 4$.

To state our results and explain our motivation, we begin by recalling the SBMSBI model (we refer to [D93] and [P02] for a general introduction to the theory of superprocesses). Let $C\left(\mathbb{R}^{d}\right)$ denote the space of continuous bounded functions on $\mathbb{R}^{d}$. We fix a constant $p>d$ and let $\phi_{p}(x):=\left(1+|x|^{2}\right)^{-p / 2}$ for $x \in \mathbb{R}^{d}$. Let $C_{p}\left(\mathbb{R}^{d}\right):=\left\{f \in C\left(\mathbb{R}^{d}\right): \sup |f(x)| / \phi_{p}(x)<\infty\right\}$. Let $M_{p}\left(\mathbb{R}^{d}\right)$ be the space of Radon measures $\mu$ on $\mathbb{R}^{d}$ such that $\langle\mu, f\rangle:=\int f(x) \mu(d x)<\infty$ for all $f \in C_{p}\left(\mathbb{R}^{d}\right)$. We endow $M_{p}\left(\mathbb{R}^{d}\right)$ with the $p$-vague topology, that is, $\mu_{k} \rightarrow \mu$ if and only if $\left\langle\mu_{k}, f\right\rangle \rightarrow\langle\mu, f\rangle$ for all $f \in C_{p}\left(\mathbb{R}^{d}\right)$. Then $M_{p}\left(\mathbb{R}^{d}\right)$ is metrizable ([186]). We denote by $\lambda$ the Lebesgue measure on $\mathbb{R}^{d}$, and note that $\lambda \in M_{p}\left(\mathbb{R}^{d}\right)$.

Let $S_{s, t}$ denote the heat semigroup in $\mathbb{R}^{d}$, that is, for $t>s$ and $f \in C\left(\mathbb{R}^{d}\right)$,

$$
S_{s, t} f(x)=\frac{1}{(2 \pi(t-s))^{d / 2}} \int_{\mathbb{R}^{d}} e^{-|y-x|^{2} / 2(t-s)} f(y) d y .
$$

We write $S_{t}:=S_{0, t}$ and $G$ for the corresponding potential operator, that is $G f=\int_{0}^{\infty} S_{t} f d t$, omitting the space variable $x$ from the notation when no confusion may occur. Given $\mu \in$

\footnotetext{
${ }^{1}$ School of Mathematical Science, Beijing Normal University, Beijing, 100875, P. R. China. Supported by Program for New Century Excellent Talents in University (NCET) and NSFC (Grant No. 10121101) . Email: wmhong@bnu.edu.cn.

${ }^{2}$ Department of Mathematics, University of Minnesota and Depts. of EE and Math., Technion. This work was partially supported by NSF grant DMS-0503775.
} 
$M_{p}\left(\mathbb{R}^{d}\right)$, a super-Brownian motion $\varrho=\left(\varrho_{t}, P_{\mu}\right)$ is an $M_{p}\left(\mathbb{R}^{d}\right)$-valued Markov process with $\varrho_{0}=\mu$ and Laplace transform given by

$$
E_{\mu} \exp \left\{-\left\langle\varrho_{t}, f\right\rangle\right\}=\exp \{-\langle\mu, v(t, \cdot)\rangle\}, \quad f \in C_{p}^{+}\left(\mathbb{R}^{d}\right),
$$

where $v(\cdot, \cdot)$ is the unique mild solution of the evolution equation

$$
\left\{\begin{array}{l}
\dot{v}(t)=\frac{1}{2} \Delta v(t)-v^{2}(t) \\
v(0)=f
\end{array}\right.
$$

and $E_{\mu}$ denotes expectation with respect to $P_{\mu}$.

Given a super-Brownian motion $\varrho=\left(\varrho_{t}, P_{\mu}\right)$ as the "environment", we will consider another super-Brownian motion with the immigration rate controlled by the trajectory of $\varrho$, the (SBMSBI) $X^{\varrho}=\left(X_{t}^{\varrho}, P_{\nu}^{\varrho}\right)$ with $X_{0}^{\varrho}=\nu$, which is again an $M_{p}\left(\mathbb{R}^{d}\right)$-valued Markov process whose quenched probability law is determined by

$$
E_{\nu}^{\varrho} \exp \left\{-\left\langle X_{t}^{\varrho}, f\right\rangle\right\}=\exp \left\{-\langle\nu, v(t, \cdot)\rangle-\int_{0}^{t}\left\langle\varrho_{s}, v(t-s, \cdot)\right\rangle d s\right\}
$$

Again, $E_{\nu}^{\varrho}$ denotes expectations with respect to $P_{\nu}^{\varrho}$.

In the following we take $\mu=\nu=\lambda$, and write $P^{\varrho}$ (resp. $P$ ) for $P_{\lambda}^{\varrho}$ (resp. $P_{\lambda}$ ). We also use $E^{\varrho}$ and $E$ for the corresponding expectations. This model was considered in [HL99] and [H02, H03, see also [DGL02, where some interesting and new phenomena were revealed under the annealed probability law:

$$
\mathbb{P}(\cdot):=\int P^{\varrho}(\cdot) P(d \varrho)
$$

with expectation denoted by $\mathbb{E}$.

Our motivation for the present study is the annealed CLT derived in [HL99], which is summarized in Theorem 1.1 below.

Theorem 1.1 (Hong-Li) Set

$$
\bar{a}_{d}(T)= \begin{cases}T^{3 / 4}, & d=3 \\ T^{1 / 2}, & d \geq 4\end{cases}
$$

and with $f \in C_{p}^{+}\left(R^{d}\right)$, define

$$
\bar{Z}_{T}^{\varrho \cdot}(f):=\bar{a}_{d}(T)^{-1}\left\{\left\langle X_{T}^{\varrho}, f\right\rangle-\mathbb{E}\left\langle X_{T}^{\varrho}, f\right\rangle\right\} .
$$

Then, $\bar{Z}_{T}^{\varrho}(f) \Rightarrow \bar{Z}_{\infty}(f)$ in distribution under the law $\mathbb{P}$ as $T \rightarrow \infty$, where $\bar{Z}_{\infty}(f)$ is a zero mean Gaussian random variable of variance

$$
\operatorname{var}\left(\bar{Z}_{\infty}(f)\right)= \begin{cases}\langle\lambda, f\rangle^{2} / 6 \pi^{3 / 2}, & d=3 \\ \langle\lambda, f\rangle^{2} / 8 \pi^{2}+\langle\lambda, f G f\rangle / 2, & d=4 \\ \langle\lambda, f G f\rangle / 2, & d \geq 5\end{cases}
$$

In particular, contrasting with the standard super Brownian motion ([186, Theorem 5.5 and Remark 6.1]), the SBMSBI exhibits smoothing of the critical dimension $d=4$, since a logarithmic term is missing in the description of the long time behavior.

In the study of motion in random media, differences exist between quenched and annealed CLT behavior, and this difference is often tied to dimension and vanishes for dimension above 
some critical value. See RS05 and Ze04 for several examples. It is thus of interest to identify whether similar behavior occurs in the case of SBMSBI. Our main result, Theorem 1.2 below, shows that this is indeed the case.

Define the centered functional $Z_{T}^{\varrho}(f)$ by

$$
Z_{T}^{\varrho \cdot}(f):=a_{d}(T)^{-1}\left\{\left\langle X_{T}^{\varrho}, f\right\rangle-E^{\varrho}\left\langle X_{T}^{\varrho}, f\right\rangle\right\}
$$

where

$$
a_{d}(T)=T^{1 / 2}, \quad d \geq 4
$$

The main result of this paper is the following.

Theorem 1.2 (Quenched CLT) Assume $d \geq 4$ and $f \in C_{p}^{+}\left(\mathbb{R}^{d}\right)$. Then, for $P$ a.e. $\varrho$, $Z_{T}^{\varrho}(f) \Rightarrow \xi(f)$ in distribution under the law $P^{\varrho}$ as $T \rightarrow \infty$, where $\xi(f)$ is a centered Gaussian variable with variance

$$
\operatorname{var}(\xi(f))=\langle\lambda, f G f\rangle / 2 .
$$

\section{Remarks}

1. As noted above, for standard SBM in the critical dimension $d=4$, it follows from I86, Remark 6.1] that the occupation measure CLT norming is $(T \log T)^{1 / 2}$.

2. In [H05], the fluctuation $b_{d}(T)^{-1}\left(E^{\varrho}\left\langle X_{T}^{\varrho}, f\right\rangle-\mathbb{E}\left\langle X_{T}^{\varrho}, f\right\rangle\right)$ between the quenched and annealed means is considered. It is shown there that the choice

$$
b_{d}(T)= \begin{cases}T^{(6-d) / 4}, & 3 \leq d \leq 5, \\ (\log T)^{1 / 2}, & d=6, \\ 1, & d \geq 7,\end{cases}
$$

leads to non-degenerate fluctuations. Comparing Theorems 1.1 and 1.2, one sees that in dimension $d=4$, the annealed fluctuations consist of quenched fluctuations (around the quenched mean) and of fluctuations of the quenched mean, and both contribute to the annealed variance. This is not the case for $d \geq 5$ : the fluctuations of the quenched mean are of lower order and wash out in the annealed CLT.

3. For $d=3$, an easy adaptation of our methods shows that the statement of Theorem 1.2 remains true with an almost sure statement being replaced by a statement in probability, that is $P^{\varrho}\left(Z_{T}^{\varrho}(f)>x\right)$ converges in probability, as $T \rightarrow \infty$, to $P(\xi(f)>x)$ for all $x$. Combined with the results in [H05], one concludes that for $d=3$, the quenched fluctuations around the quenched mean are of lower order than the fluctuations of the quenched mean. Together with Theorems 1.1 and 1.2, this gives a fairly complete description of the CLT in all dimensions $d \geq 3$.

4. A functional version of Theorem 1.2 can be derived by using similar ideas. We prefer to bring here the shorter proof for the standard CLT.

5. Large deviations for this and related processes were studied in [IL93], [L93] and [LR95]. 


\section{Proof of Theorem 1.2}

Set $d \geq 4$ and $f_{t}:=a_{d}(t)^{-1} f$ with $f \in C_{p}^{+}\left(\mathbb{R}^{d}\right)$. For each fixed $t$, the mild form $v_{t}(r, x)$ of equation (1.2) with $v_{t}(0, x)=f_{t}(x)$ is

$$
v_{t}(r, x)=S_{r} f_{t}(x)-\int_{0}^{r} S_{r-h} v_{t}(h, \cdot)^{2}(x) d h, \quad 0 \leq r \leq t .
$$

From equations (1.3) and (2.1), it follows that

$$
E^{\varrho}\left\langle X_{t}^{\varrho}, f_{t}\right\rangle=\left\langle\lambda, S_{t} f_{t}\right\rangle+\int_{0}^{t}\left\langle\varrho_{s}, S_{t-s} f_{t}\right\rangle d s
$$

Combined with (1.4), we get

$$
E^{\varrho} \exp \left\{-Z_{t}^{\varrho}(f)\right\}=\exp \left\{\left\langle\lambda, \int_{0}^{t} S_{s} v_{t}^{2}(t-s, \cdot) d s\right\rangle+\int_{0}^{t}\left\langle\varrho_{s}, \int_{0}^{t-s} S_{r} v_{t}^{2}(t-s-r, \cdot) d r\right\rangle d s\right\} .
$$

The proof of Theorem 1.2 builds upon the following two propositions. The proof of Proposition 2.2 will take up most of our effort.

Proposition 2.1 With the above notation,

$$
\left\langle\lambda, \int_{0}^{t} S_{s} v_{t}^{2}(t-s, \cdot) d s\right\rangle \longrightarrow_{t \rightarrow \infty} 0 .
$$

Set $g_{t}(u, x)=\int_{0}^{u} S_{r} v_{t}^{2}(u-r, \cdot)(x) d r=\int_{0}^{u} S_{u-r} v_{t}^{2}(r, \cdot)(x) d r$.

Proposition 2.2 For P-a.e. $\varrho$,

$$
\Gamma(t):=\int_{0}^{t}\left\langle\varrho_{s}, g_{t}(t-s)\right\rangle d s \longrightarrow_{t \rightarrow \infty}\langle\lambda, f G f\rangle / 2 .
$$

Proof of Theorem 1.2 The theorem is an immediate consequence of (2.2), Proposition 2.1 and Proposition 2.2.

Proof of Proposition 2.1 A direct computation shows that, for any $d \geq 3$,

$$
\int_{0}^{\infty} d s\left\langle\lambda, f S_{2 s} f\right\rangle<\infty
$$

From (2.1), it follows that

$$
\left\langle\lambda, \int_{0}^{t} S_{s} v_{t}^{2}(t-s, \cdot) d s\right\rangle=\left\langle\lambda, \int_{0}^{t} v_{t}^{2}(t-s, \cdot) d s\right\rangle \leq \int_{0}^{t}\left\langle\lambda,\left(S_{t-s} f_{t}\right)^{2}\right\rangle d s=t^{-1} \int_{0}^{t} d s\left\langle\lambda, f S_{2 s} f\right\rangle .
$$

Using (2.3), the result follows.

Proof of Proposition 2.2 We recall from [186, Theorem 3.2] that for any $C_{p}\left(R^{d}\right)^{+}$-valued continuous path $F(s)$, the Laplace transform of $\int_{0}^{t}\left\langle\varrho_{s}, F(t-s)\right\rangle d s$ is given by

$$
E \exp \left\{-\theta \int_{0}^{t}\left\langle\varrho_{s}, F(t-s)\right\rangle d s\right\}=\exp \{-\langle\lambda, u(t, \theta ; \cdot)\rangle\}, \theta>0
$$


where $u(s, \theta ; x)$ is the nonnegative solution of the following mild equation

$$
u(s, \theta ; x)=\theta \int_{0}^{s} S_{s-r} F(r)(x) d r-\int_{0}^{s} S_{s-r} u^{2}(r, \theta)(x) d r, \quad 0 \leq s \leq t .
$$

(In fact, (2.4) and (2.5) hold true for $|\theta|<c$ for $c$ a small enough constant, see [H03.) Differentiating with respect to $\theta$ in (2.4) and (2.5), we obtain

$$
E\left[\int_{0}^{t}\left\langle\varrho_{s}, F(t-s)\right\rangle d s\right]=\left\langle\lambda, \int_{0}^{t} S_{t-s} F(s) d s\right\rangle=\int_{0}^{t}\langle\lambda, F(s)\rangle d s
$$

where the invariance of $\lambda$ under shifts was used in the second equality. Similarly,

$$
\operatorname{var}\left[\int_{0}^{t}\left\langle\varrho_{s}, F(t-s)\right\rangle d s\right]=2 \int_{0}^{t}\left\langle\lambda,\left(\int_{0}^{s} S_{s-r} F(r) d r\right)^{2}\right\rangle d s .
$$

In the sequel, we let $A$ denote a constant whose value may change from line to line and which may depend on the dimension and on $f$, but not on $s, t, x$, etc. Let us recall the useful estimate

$$
\left\|S_{s} f\right\| \leq A \cdot\left(1 \wedge s^{-d / 2}\right)
$$

where $\|\cdot\|$ denotes the supremum norm.

Lemma 2.1 Let $F(s)=g_{t}(s)$. Then,

$$
E\left[\int_{0}^{t}\left\langle\varrho_{s}, g_{t}(t-s)\right\rangle d s\right] \longrightarrow_{t \rightarrow \infty}\langle\lambda, f G f\rangle / 2 .
$$

Proof. Note that

$$
\begin{aligned}
E\left[\int_{0}^{t}\left\langle\varrho_{s}, g_{t}(t-s)\right\rangle d s\right] & =\int_{0}^{t} d s \int_{0}^{s}\left\langle\lambda, v_{t}^{2}(r, \cdot)\right\rangle d r \\
& =\int_{0}^{t} d s \int_{0}^{s}\left\langle\lambda,\left(S_{r} f_{t}\right)^{2}\right\rangle d r-\int_{0}^{t} d s \int_{0}^{s}\left\langle\lambda,\left(S_{r} f_{t}\right)^{2}-v_{t}^{2}(r, \cdot)\right\rangle d r .
\end{aligned}
$$

One has

$$
\left.\int_{0}^{t} d s \int_{0}^{s}\left\langle\lambda,\left(S_{r} f_{t}\right)^{2}\right\rangle d r=t^{-1} \int_{0}^{t} d s \int_{0}^{s}\left\langle\lambda, f S_{2 s} f\right)\right\rangle d r \longrightarrow\langle\lambda, f G f\rangle / 2 .
$$

On the other hand, by (2.1),

$$
\begin{aligned}
\int_{0}^{t} d s \int_{0}^{s}\left\langle\lambda,\left(S_{r} f_{t}\right)^{2}-v_{t}^{2}(r, \cdot)\right\rangle d r & \leq 2 \int_{0}^{t} d s \int_{0}^{s}\left\langle\lambda, S_{r} f_{t} \cdot \int_{0}^{r} S_{r-h} v_{t}^{2}(h) d h\right\rangle d r \\
& \leq 2 \int_{0}^{t} d s \int_{0}^{s}\left\langle\lambda, S_{r} f_{t} \cdot \int_{0}^{r} S_{r-h}\left(S_{h} f_{t}\right)^{2} d h\right\rangle d r \\
& \leq A t^{-3 / 2} \int_{0}^{t} d s \int_{0}^{s}\left\langle\lambda,\left(S_{r} f\right)^{2}\right\rangle d r \cdot \int_{0}^{t}\left(1 \wedge h^{-d / 2}\right) d h \\
& =A t^{-3 / 2} \int_{0}^{t} d s \int_{0}^{s}\left\langle\lambda, f S_{2 r} f\right\rangle d r \cdot \int_{0}^{t}\left(1 \wedge h^{-d / 2}\right) d h \\
& \leq \frac{A}{\sqrt{t}} \cdot \frac{1}{t} \int_{0}^{t} d s \int_{0}^{\infty}\left\langle\lambda, f S_{2 r} f\right\rangle d r
\end{aligned}
$$


which goes to 0 when $d \geq 3$ as $t \rightarrow \infty$ due to (2.3); here, we used (2.8) at the third inequality. Substituting in (2.6), the lemma follows.

We return to the proof of Proposition 2.2. In view of Lemma 2.1, it is enough to prove that $\Gamma(t)-E \Gamma(t) \rightarrow 0$, as $t \rightarrow \infty$, i.e.,

$$
\int_{0}^{t}\left\langle\varrho_{s}, g_{t}(t-s)\right\rangle d s-E\left[\int_{0}^{t}\left\langle\varrho_{s}, g_{t}(t-s)\right\rangle d s\right] \rightarrow 0, P \text { a.s. } .
$$

For any $n$ integer and $n \leq t_{1} \leq t \leq n+1$, let $\delta=t-t_{1}$, and set $\Delta \Gamma\left(t_{1}, t\right):=\Gamma(t)-\Gamma\left(t_{1}\right)$. Write $\left.\Delta \Gamma\left(t_{1}, t\right)\right)$ as the sum of four terms

$$
\Delta \Gamma\left(t_{1}, t\right)=\Delta \Gamma_{1}\left(t_{1}, t\right)+\Delta \Gamma_{2}\left(t_{1}, t\right)+\Delta \Gamma_{3}\left(t_{1}, t\right)+\Delta \Gamma_{4}\left(t_{1}, t\right)
$$

where

$$
\begin{aligned}
\Delta \Gamma_{1}\left(t_{1}, t\right) & :=\int_{t_{1}}^{t}\left\langle\varrho_{r}, \int_{0}^{t-r} S_{t-r-h} v_{t}^{2}(h) d h\right\rangle d r, \\
\Delta \Gamma_{2}\left(t_{1}, t\right) & :=\int_{0}^{t_{1}}\left\langle\varrho_{r}, \int_{t_{1}-r}^{t-r} S_{t-r-h} v_{t}^{2}(h) d h\right\rangle d r, \\
\Delta \Gamma_{3}\left(t_{1}, t\right) & :=\int_{0}^{t_{1}}\left\langle\varrho_{r}, \int_{0}^{t_{1}-r} S_{t-r-h}\left[v_{t}^{2}(h)-v_{t_{1}}^{2}(h)\right] d h\right\rangle d r, \\
\Delta \Gamma_{4}\left(t_{1}, t\right) & :=\int_{0}^{t_{1}}\left\langle\varrho_{r}, \int_{0}^{t_{1}-r}\left[S_{t-r-h}-S_{t_{1}-r-h}\right] v_{t_{1}}^{2}(h) d h\right\rangle d r .
\end{aligned}
$$

We estimate separately the moments of centered versions of $\Delta \Gamma_{i}\left(t_{1}, t\right)$.

\section{Lemma 2.2}

$$
\operatorname{var}\left[\Delta \Gamma_{1}\left(t_{1}, t\right)\right] \leq A \delta^{2} n^{-2} .
$$

Proof. Recall that $\Delta \Gamma_{1}\left(t_{1}, t\right)=\int_{t_{1}}^{t}\left\langle\varrho_{r}, g_{t}(t-r)\right\rangle d r$. We have, again from [I86, Theorem 3.2], that for $\theta \geq 0$,

$$
\begin{aligned}
E \exp \left\{-\theta \int_{t_{1}}^{t}\left\langle\varrho_{r}, g_{t}(t-r)\right\rangle d r\right\} & =E \exp \left\{-\left\langle\varrho_{t_{1}}, u\left(t_{1}, t, \theta ; \cdot\right)\right\rangle\right\} \\
& =\exp \left\{-\left\langle\lambda, w\left(0, t_{1}, \theta ; \cdot\right)\right\rangle,\right\}
\end{aligned}
$$

where $u(s, t, \theta ; \cdot)$ is the nonnegative solution of the following mild equation

$$
u(s, t, \theta ; x)=\theta \int_{s}^{t} S_{s, r} g_{t}(t-r)(x) d r-\int_{s}^{t} S_{s, r} u^{2}(r, t, \theta)(x) d r, \quad t_{1} \leq s \leq t,
$$

and $w\left(s, t_{1}, \theta ; x\right)$ is the nonnegative solution of the following mild equation

$$
w\left(s, t_{1}, \theta ; x\right)=S_{s, t_{1}} u\left(t_{1}, t, \theta ; \cdot\right)(x)-\int_{s}^{t_{1}} S_{s, r} w^{2}\left(r, t_{1}, \theta\right)(x) d r, \quad 0 \leq s \leq t_{1} .
$$

Obviously,

$$
\operatorname{var}\left[\Delta \Gamma_{1}\left(t_{1}, t\right)\right]=-\left.\frac{\partial^{2}\left\langle\lambda, w\left(0, t_{1}, \theta ; \cdot\right)\right\rangle}{\partial \theta^{2}}\right|_{\theta=0}
$$


Performing the differentiation and using that $\left.u\right|_{\theta=0}=\left.w\right|_{\theta=0}=0$, we obtain

$$
\begin{aligned}
& \operatorname{var}\left[\Delta \Gamma_{1}\left(t_{1}, t\right)\right] \\
= & 2\left\langle\lambda, \int_{t_{1}}^{t} S_{0, s}\left[\int_{s}^{t} S_{s, r} g_{t}(t-r) d r\right]^{2} d s\right\rangle+2\left\langle\lambda, \int_{0}^{t_{1}} S_{0, s}\left[\int_{t_{1}}^{t} S_{s, r} g_{t}(t-r) d r\right]^{2} d s\right\rangle \\
\leq & 2 t^{-2}\left[\left\langle\lambda, \int_{t_{1}}^{t} S_{s}\left[\int_{s}^{t} \int_{0}^{t-r} S_{t-s-l}\left(S_{l} f\right)^{2} d l d r\right]^{2} d s\right\rangle+\left\langle\lambda, \int_{0}^{t_{1}} S_{s}\left[\int_{t_{1}}^{t} \int_{0}^{t-r} S_{t-s-l}\left(S_{l} f\right)^{2} d l d r\right]^{2} d s\right\rangle\right] \\
\leq & A t^{-2}\left[\left\langle\lambda, \int_{t_{1}}^{t}\left[(t-s) S_{t-s} f\right]^{2} d s\right\rangle+\left\langle\lambda, \int_{0}^{t_{1}}\left[\int_{t_{1}}^{t} S_{t-s} f d r\right]^{2} d s\right\rangle\right] \\
\leq & A n^{-2} \delta^{2},
\end{aligned}
$$

in which (2.8) has been used several times.

Lemma 2.3 With the above notation,

$$
E\left[\Delta \Gamma_{2}\left(t_{1}, t\right)-E \Delta \Gamma_{2}\left(t_{1}, t\right)\right]^{4} \leq A \delta^{2} n^{-2} .
$$

Proof. We have $\Delta \Gamma_{2}\left(t_{1}, t\right)=\int_{0}^{t_{1}}\left\langle\varrho_{r}, F_{t}\left(t_{1}-r\right)\right\rangle d r$ where $F_{t}(r):=\int_{r}^{\delta+r} S_{\delta+r-l} v_{t}^{2}(l) d l$, then $\Delta \Gamma_{2}\left(t_{1}, t\right)=\int_{0}^{t_{1}}\left\langle\varrho_{r}, F\left(t_{1}-r\right)\right\rangle d r$. Let

$$
u^{(i)}(r, x):=\left.\frac{\partial^{i} u(r, x, \theta)}{\partial \theta^{i}}\right|_{\theta=0}, i=1,2,3 .
$$

Differentiating with respect to $\theta$ in (2.4) and (2.5), and using again that $\left.u\right|_{\theta=0}=0$, we obtain

$$
\begin{aligned}
& E\left[\Delta \Gamma_{2}\left(t_{1}, t\right)-E \Delta \Gamma_{2}\left(t_{1}, t\right)\right]^{4} \\
= & 3\left(\int_{0}^{t_{1}}\left\langle\lambda, u^{(1)}(r)^{2}\right\rangle d r\right)^{2}+3 \int_{0}^{t_{1}}\left\langle\lambda, u^{(2)}(r)^{2}\right\rangle d r+4 \int_{0}^{t_{1}}\left\langle\lambda, u^{(1)}(r) u^{(3)}(r)\right\rangle d r \\
:= & 3 I^{2}+3 J+4 K,
\end{aligned}
$$

where for $0 \leq r \leq t_{1}$,

$$
\begin{aligned}
u^{(1)}(r, x) & =\int_{0}^{r} S_{r-s} F_{t}(s) d s=\int_{0}^{r} S_{r-s} \int_{s}^{\delta+s} S_{\delta+s-l} v_{t}^{2}(l) d l d s \\
& \leq A t_{1}^{-1} \int_{0}^{r} S_{r-s} \int_{s}^{\delta+s} S_{\delta+s-l}\left(S_{l} f\right)^{2} d l d s \\
& \leq A t_{1}^{-1} \delta \cdot r S_{\delta+r} f \\
\left|u^{(2)}(r, x)\right| & =\left|-2 \int_{0}^{r} S_{r-s} u^{\prime}(s)^{2} d s\right| \leq A t_{1}^{-2} \delta^{2} \cdot \int_{0}^{r} S_{r-s}\left(s S_{\delta+s} f\right)^{2} d s \\
& \leq A t_{1}^{-2} \delta^{2} \cdot r S_{\delta+r} f \\
\left|u^{(3)}(r, x)\right| & =\left|-6 \int_{0}^{r} S_{r-s} u^{\prime}(s) u^{\prime \prime}(s) d s\right| \leq A t_{1}^{-3} \delta^{3} \cdot \int_{0}^{r} S_{r-s}\left(s S_{\delta+s} f\right)^{2} d s \\
& \leq A t_{1}^{-3} \delta^{3} \cdot r S_{\delta+r} f .
\end{aligned}
$$


Thus, we obtain, using that $d \geq 4$,

$$
I \leq A t_{1}^{-2} \delta^{2} \cdot \int_{0}^{t_{1}}\left\langle\lambda,\left(r S_{\delta+r} f\right)^{2}\right\rangle d r \leq A t_{1}^{-2} \delta^{2} \cdot \int_{0}^{t_{1}} r^{2}\left(1 \wedge(\delta+r)^{-d / 2}\right) d r \leq A t_{1}^{-1} \delta^{2} .
$$

Similarly, we have

$$
J=\int_{0}^{t_{1}}\left\langle\lambda, u^{(2)}(r)^{2}\right\rangle d r \leq A t_{1}^{-4} \delta^{4} \cdot \int_{0}^{t_{1}}\left\langle\lambda,\left(r S_{\delta+r} f\right)^{2}\right\rangle d r \leq A t_{1}^{-3} \delta^{4}
$$

and

$$
K=\int_{0}^{t_{1}}\left\langle\lambda, u^{(1)}(r) u^{(3)}(r)\right\rangle d r \leq A t_{1}^{-4} \delta^{4} \cdot \int_{0}^{t_{1}}\left\langle\lambda,\left(r S_{\delta+r} f\right)^{2}\right\rangle d r \leq A t_{1}^{-3} \delta^{4}
$$

Substituting in (2.11) completes the proof.

Lemma 2.4 With the above notation,

$$
\operatorname{var}\left[\Delta \Gamma_{3}\left(t_{1}, t\right)\right] \leq A \delta^{2} n^{-3}
$$

Proof. We begin by considering the difference $v_{t}(r, x)-v_{t_{1}}(r, x)$. From (2.1), we have

$$
v_{t}(r, x)-v_{t_{1}}(r, x)=S_{r} f_{t}(x)-S_{r} f_{t_{1}}(x)-\int_{0}^{r} S_{r-h}\left[v_{t}^{2}(h, \cdot)-v_{t_{1}}^{2}(h, \cdot)\right](x) d h .
$$

A direct computation reveals that $\left\|S_{r} f_{t}-S_{r} f_{t_{1}}\right\| \leq A \delta t_{1}^{-3 / 2}\left(1 \wedge r^{-d / 2}\right)$. Since $v_{t}(r, x) \leq S_{r} f_{t}$, it follows that $\left\|S_{r-h}\left[v_{t}^{2}(h, \cdot)-v_{t_{1}}^{2}(h, \cdot)\right]\right\| \leq\left\|2\left[v_{t}(h, \cdot)-v_{t_{1}}(h, \cdot)\right] S_{r} f_{t_{1}}\right\| \leq A t_{1}^{-1 / 2}\left(1 \wedge r^{-d / 2}\right) \| v_{t}(h, \cdot)-$ $v_{t_{1}}(h, \cdot) \|$. Thus from (2.12) we get,

$$
\left\|v_{t}(r, \cdot)-v_{t_{1}}(r, \cdot)\right\| \leq A \delta t_{1}^{-3 / 2}\left(1 \wedge r^{-d / 2}\right)+A t_{1}^{-1 / 2}\left(1 \wedge r^{-d / 2}\right) \int_{0}^{r}\left\|v_{t}(h, \cdot)-v_{t_{1}}(h, \cdot)\right\| d h .
$$

Writing $a_{r}=\left\|v_{t}(r, \cdot)-v_{t_{1}}(r, \cdot)\right\| \geq 0, b_{r}=A \delta t_{1}^{-3 / 2}\left(1 \wedge r^{-d / 2}\right) \geq 0$ and $c_{r}=A t_{1}^{-1 / 2}\left(1 \wedge r^{-d / 2}\right) \geq 0$, we thus have

$$
a_{r} \leq b_{r}+c_{r} \int_{0}^{r} a_{s} d s
$$

By a version of Gronwall's inequality,

$$
a_{r} \leq b_{r}+c_{r} \int_{0}^{r} e^{\int_{s}^{t} c_{u} d u} b_{s} d s .
$$

(This can be seen by setting $z_{r}=\int_{0}^{r} a_{s} d s$ and noting that $z_{r}$ satisfies the differential inequality $d z_{r} / d r \leq b_{r}+c_{r} z_{r}$, with $z_{0}=0$.) Thus,

$$
\begin{aligned}
& \left\|v_{t}(r, \cdot)-v_{t_{1}}(r, \cdot)\right\| \\
\leq & A \delta t_{1}^{-3 / 2}\left(1 \wedge r^{-d / 2}\right)+A^{2} t_{1}^{-1 / 2}\left(1 \wedge r^{-d / 2}\right) \int_{0}^{r} \delta t_{1}^{-3 / 2}\left(1 \wedge s^{-d / 2}\right) \exp \left\{A t_{1}^{-1 / 2} \int_{s}^{r}\left(1 \wedge u^{-d / 2}\right) d u\right\} d s \\
\leq & A \delta t_{1}^{-1}\left(1 \wedge r^{-d / 2}\right) .
\end{aligned}
$$


Once more by (2.12) we have

$$
\begin{aligned}
& \left|v_{t}(r, x)-v_{t_{1}}(r, x)\right| \\
\leq & \left|S_{r} f_{t}(x)-S_{r} f_{t_{1}}(x)\right|+\int_{0}^{r}\left\|v_{t}(h, \cdot)-v_{t_{1}}(h, \cdot)\right\| \cdot\left|S_{r-h}\left[v_{t}(h, \cdot)+v_{t_{1}}(h, \cdot)\right](x)\right| d h \\
\leq & \delta t_{1}^{-3 / 2} S_{r} f(x)+A \delta t_{1}^{-3 / 2} S_{r} f(x) \cdot \int_{0}^{r}\left(1 \wedge h^{-d / 2}\right) d h \\
\leq & A \delta t_{1}^{-3 / 2} S_{r} f(x) .
\end{aligned}
$$

Now we can estimate the variance of $\Gamma_{3}\left(t_{1}, t\right)$. By (2.7) with $F(r)=\int_{0}^{r} S_{t-t_{1}+r-l}\left[v_{t}^{2}(l)-v_{t_{1}}^{2}(l)\right] d l$,

$$
\begin{aligned}
\operatorname{var}\left[\Delta \Gamma_{3}\left(t_{1}, t\right)\right] & =2 \int_{0}^{t_{1}}\left\langle\lambda,\left[\int_{0}^{r} S_{r-h} \int_{0}^{h} S_{t-t_{1}+h-l}\left[v_{t}^{2}(l)-v_{t_{1}}^{2}(l)\right] d l d h\right]^{2}\right\rangle d r \\
& \leq A \int_{0}^{t_{1}}\left\langle\lambda,\left[\int_{0}^{r} \int_{0}^{h} S_{\delta+r-l}\left|v_{t}(l)-v_{t_{1}}(l)\right|\left|v_{t}(l)+v_{t_{1}}(l)\right| d l d h\right]^{2}\right\rangle d r \\
& \leq A \delta^{2} t_{1}^{-4} \int_{0}^{t_{1}} r^{2}\left[1 \wedge(r+\delta)^{-d / 2}\right] d r \\
& \leq A \delta^{2} n^{-3} .
\end{aligned}
$$

This completes the proof of the lemma.

Before providing an estimate on the moments of $\Delta \Gamma_{4}\left(t_{1}, t\right)$, we need an a-priori simple estimate on time differences of the heat kernel $p(t, x, y)=(2 \pi t)^{-d / 2} \exp \left(-|x-y|^{2} / 2 t\right)$. Since we did not find a direct reference for it, we provide the proof.

Lemma 2.5 There is a constant $A$ such that for any $t \geq \tau>0$, we have

$$
\sup _{0<s \leq \tau \leq t} s^{-1}|p(t+s, x, y)-p(t, x, y)| \leq A \tau^{-1}[p(t+2 \tau, x, y)+p(t, x, y)],
$$

Proof. Consider first $\tau=1$. Let $z=|x-y|$, two cases should be considered:

Case 1: $z^{2}<2 d(t+1)$. Note that

$$
|p(t+s, x, y)-p(t, x, y)|=p(t, x, y)\left|\exp \left\{\frac{z^{2} s}{2 t(t+s)}\right\}\left(\frac{t}{t+s}\right)^{d / 2}-1\right|
$$

and $\exp \left\{\frac{z^{2} s}{2 t(t+s)}\right\}=1+s R_{1}(s, t, z),\left(\frac{t}{t+s}\right)^{d / 2}=\left(1-\frac{s}{t+s}\right)^{d / 2}=1+s R_{2}(s, t, z)$, where $R_{1}(s, t, z)$, $R_{2}(s, t, z)$ are bounded by a constant when $z^{2}<2 d(t+1), 0<s \leq 1 \leq t$. Thus, we get

$$
\sup _{0<s \leq 1} \sup _{z^{2}<2 d(t+1)} s^{-1}|p(t+s, x, y)-p(t, x, y)| \leq A p(t, x, y) .
$$

Case 2: $z^{2} \geq 2 d(t+1)$. Since $\frac{\partial}{\partial t} p(t, z)=p(t, z)\left[-\frac{d}{2 t}+\frac{z^{2}}{2 t^{2}}\right]$,

$$
\begin{aligned}
|p(t+s, z)-p(t, z)| & =\left|\int_{0}^{s} p(t+u, z)\left[-\frac{d}{2(t+u)}+\frac{z^{2}}{2(t+u)^{2}}\right] d u\right| \\
& \leq \int_{0}^{s} p(t+u, z) \frac{z^{2}}{2(t+u)^{2}} d u
\end{aligned}
$$


where the inequality uses that $\left|-\frac{d}{2(t+u)}+\frac{z^{2}}{2(t+u)^{2}}\right| \leq \frac{z^{2}}{2(t+u)^{2}}$ when $z^{2} \geq 2 d(t+1)$. But

$$
\begin{aligned}
\frac{z^{2}}{2(t+u)^{2}} p(t+u, z) & =p(t+2, z)\left(\frac{t+2}{t+u}\right)^{d / 2} \frac{z^{2}}{(t+u)^{2}} \exp \left\{-\frac{z^{2}}{2}\left[\frac{2-u}{(t+u)(t+2)}\right]\right\} \\
& \leq A \cdot p(t+2, z) \frac{z^{2}}{(t+u)^{2}} \exp \left\{-\frac{z^{2}}{2}\left[\frac{2-u}{(t+u)(t+2)}\right]\right\}
\end{aligned}
$$

(note that $0<u \leq s \leq 1 \leq t$ ) and

$$
\sup _{z^{2} \geq 2 d(t+1)} \frac{z^{2}}{(t+u)^{2}} \exp \left\{-\frac{z^{2}}{2}\left[\frac{2-u}{(t+u)(t+2)}\right]\right\}<\infty .
$$

So

$$
\sup _{0<s \leq 1} \sup _{z^{2} \geq 2 d(t+1)} s^{-1}|p(t+s, x, y)-p(t, x, y)| \leq A p(t+2, x, y) .
$$

Combining (2.14) and (2.15) we obtain (2.13) when $\tau=1$. For general $\tau>0$, we use the scaling properties of $p(t, z)$. We have

$$
\begin{aligned}
s^{-1}|p(t+s, z)-p(t, z)| & =\tau^{-d / 2} s^{-1}\left|p\left(\tau^{-1}(t+s), \tau^{-1 / 2} z\right)-p\left(\tau^{-1} t, \tau^{-1 / 2} z\right)\right| \\
& =\tau^{-d / 2} \tau^{-1}\left[\tau s^{-1}\left|p\left(\tau^{-1}(t+s), \tau^{-1 / 2} z\right)-p\left(\tau^{-1} t, \tau^{-1 / 2} z\right)\right|\right] \\
& \left.\leq A \tau^{-d / 2} \tau^{-1}\left[p\left(\tau^{-1} t+2\right), \tau^{-1 / 2} z\right)+p\left(\tau^{-1} t, \tau^{-1 / 2} z\right)\right] \\
& \left.\leq A \tau^{-1}[p(t+2 \tau), z)+p(t, z)\right],
\end{aligned}
$$

where the third inequality follows from the case already considered because $0<\tau^{-1} s \leq 1 \leq \tau^{-1} t$. This complete the proof.

Lemma 2.6 With the notation above, we have

$$
E\left[\Delta \Gamma_{4}\left(t_{1}, t\right)-E \Delta \Gamma_{4}\left(t_{1}, t\right)\right]^{4} \leq A \delta^{2} n^{-2}
$$

Proof. The formula for the fourth moment of $\Delta \Gamma_{4}\left(t_{1}, t\right)$ is as in the proof of Lemma 2.3 , except that the function $F_{t}(r)$ is replaced by the function $\tilde{F}_{t_{1}}(r):=\int_{0}^{r}\left[S_{\delta+r-l}-S_{r-l}\right] v_{t_{1}}^{2}(l) d l$, and $\Delta \Gamma_{4}\left(t_{1}, t\right)=\int_{0}^{t_{1}}\left\langle\lambda, \tilde{F}_{t_{1}}(r)\right\rangle d r$. Recalling that $\delta=t-t_{1}$, we obtain for $0 \leq r \leq t_{1}$ that,

$$
\begin{aligned}
\left|u^{(1)}(r, x)\right| & =\left|\int_{0}^{r} S_{r-s} \tilde{F}_{t_{1}}(s) d s\right|=\left|\int_{0}^{r} S_{r-s} \int_{0}^{s}\left[S_{\delta+s-l}-S_{s-l}\right] v_{t_{1}}^{2}(l) d l d s\right| \\
& \leq \int_{0}^{r} l\left|\left[S_{\delta+l}-S_{l}\right] v_{t_{1}}^{2}(r-l)\right| d l \\
& \leq \int_{0}^{\delta} l\left[S_{\delta+l}+S_{l}\right]\left(S_{(r-l)} f_{t_{1}}\right)^{2} d l+A \int_{\delta}^{r} \delta\left[S_{3 l}+S_{l}\right]\left(S_{(r-l)} f_{t_{1}}\right)^{2} d l \\
& \leq A \delta t_{1}^{-1}\left[\delta\left(S_{\delta+r}+S_{r}\right) f+\int_{\delta}^{r}\left(S_{r+2 l}+S_{r}\right) f \cdot\left(1 \wedge(r-l)^{-d / 2}\right) d l\right]
\end{aligned}
$$


where the third step is from Lemma 2.5 (with $\tau=t=l, s=\delta$ there). By a similar calculation we get

$$
\begin{aligned}
\left|u^{(2)}(r, x)\right| & =\left|-2 \int_{0}^{r} S_{r-s} u^{(1)}(s)^{2} d s\right| \\
& \leq A t_{1}^{-2} \delta^{2} \cdot\left[\delta\left(S_{\delta+r}+S_{r}\right) f+\int_{0}^{r} d s \int_{\delta}^{s}\left(S_{r+2 l}+S_{r}\right) f \cdot\left(1 \wedge(s-l)^{-d / 2}\right) d l\right], \\
\left|u^{(3)}(r, x)\right| & =\left|-6 \int_{0}^{r} S_{r-s} u^{(1)}(s) u^{(2)}(s) d s\right| \\
& \leq A t_{1}^{-3} \delta^{3} \cdot\left[\delta\left(2 r^{3 / 2}+\delta\right)\left(S_{\delta+r}+S_{r}\right) f+r^{1 / 2} \int_{0}^{r} d s \int_{\delta}^{s}\left(S_{r+2 l}+S_{r}\right) f \cdot\left(1 \wedge(s-l)^{-d / 2}\right) d l\right],
\end{aligned}
$$

and the estimate (2.8) was used many times. Then

$$
\begin{aligned}
I & =\int_{0}^{t_{1}}\left\langle\lambda, u^{(1)}(r)^{2}\right\rangle d r \\
& \leq A \delta^{2} t_{1}^{-2} \int_{0}^{t_{1}}\left\langle\lambda,\left[\delta\left(S_{\delta+r}+S_{r}\right) f+\int_{\delta}^{r}\left(S_{r+2 l}+S_{r}\right) f \cdot\left(1 \wedge(r-l)^{-d / 2}\right) d l\right]^{2}\right\rangle d r \\
& \leq A \delta^{2} t_{1}^{-1}
\end{aligned}
$$

and $J=3 \int_{0}^{t_{1}}\left\langle\lambda, u^{(2)}(r)^{2}\right\rangle d r \leq A \delta^{4} t_{1}^{-2}, K=\int_{0}^{t_{1}}\left\langle\lambda, u^{(1)}(r) u^{(3)}(r)\right\rangle d r \leq A \delta^{4} t_{1}^{-2}$. So

$$
E\left[\Delta \Gamma_{4}\left(t_{1}, t\right)-E \Delta \Gamma_{4}\left(t_{1}, t\right)\right]^{4}=3 I^{2}+3 J+4 K \leq A \delta^{4} n^{-2},
$$

which completes the proof.

We return to the proof of Proposition 2.2, Let $\bar{\Gamma}(t):=\Gamma(t)-E \Gamma(t)$ denote the centered $\Gamma(t)$, and define $\Delta \overline{\Gamma_{i}}$ similarly. For any $\varepsilon>0$ and $\alpha \in(1,4 / 3)$,

$$
\begin{aligned}
& P\left(\max _{n^{\alpha} \leq t \leq(n+1)^{\alpha}}\left|\bar{\Gamma}(t)-\bar{\Gamma}\left(n^{\alpha}\right)\right|>\varepsilon\right) \\
\leq & \sum_{k=1}^{\infty} P\left(\max _{0 \leq j \leq 2 \alpha n^{\alpha-1} 2^{k}}\left|\bar{\Gamma}\left(n^{\alpha}+2^{-k}(j+1)\right)-\bar{\Gamma}\left(n^{\alpha}+2^{-k} j\right)\right|>\frac{\epsilon}{4 \alpha n^{\alpha-1} k^{2}}\right) \\
= & \sum_{k=1}^{\infty} P\left(\max _{0 \leq j \leq 2 \alpha n^{\alpha-1} 2^{k}}\left|\Delta \bar{\Gamma}\left(n^{\alpha}+2^{-k} j, n^{\alpha}+2^{-k}(j+1)\right)\right|>\frac{\epsilon}{4 \alpha n^{\alpha-1} k^{2}}\right) \\
\leq & \sum_{k=1}^{\infty} \sum_{i=1}^{4} 2 \alpha n^{\alpha-1} 2^{k} \max _{0 \leq j \leq 2 \alpha n^{\alpha-1} 2^{k}} P\left(\left|\Delta \overline{\Gamma_{i}}\left(n^{\alpha}+2^{-k} j, n^{\alpha}+2^{-k}(j+1)\right)\right|>\frac{\epsilon}{16 \alpha n^{\alpha-1} k^{2}}\right) .
\end{aligned}
$$

By Chebyshev's inequality and Lemmas 2.2 and 2.4, for $i=1,3$,

$$
P\left(\left|\Delta \overline{\Gamma_{i}}\left(n^{\alpha}+2^{-k} j, n^{\alpha}+2^{-k}(j+1)\right)\right|>\frac{\epsilon}{16 \alpha n^{\alpha-1} k^{2}}\right) \leq A_{\alpha} \epsilon^{-2} k^{4} 2^{-2 k} n^{-2} .
$$

Similarly, using Lemmas 2.3 and 2.6, we obtain for $i=2,4$,

$$
P\left(\left|\Delta \overline{\Gamma_{i}}\left(n^{\alpha}+2^{-k} j, n^{\alpha}+2^{-k}(j+1)\right)\right|>\frac{\epsilon}{16 \alpha n^{\alpha-1} k^{2}}\right) \leq A_{\alpha} \epsilon^{-4} k^{8} 2^{-2 k} n^{2 \alpha-4} .
$$


Thus, adjusting the value of $A_{\alpha}$, using that $2 \alpha-4>-2$,

$$
P\left(\max _{n^{\alpha} \leq t \leq(n+1)^{\alpha}}\left|\bar{\Gamma}(t)-\bar{\Gamma}\left(n^{\alpha}\right)\right|>\varepsilon\right) \leq A_{\alpha} \varepsilon^{-4} n^{3 \alpha-5} \sum_{k=1}^{\infty} k^{8} 2^{-k} \leq A_{\alpha} \varepsilon^{-4} n^{3 \alpha-5} .
$$

By the Borel-Cantelli Lemma, we get $\max _{n^{\alpha} \leq t \leq(n+1)^{\alpha}}\left|\bar{\Gamma}(t)-\bar{\Gamma}\left(n^{\alpha}\right)\right| \rightarrow 0, P-$ a.s.. Thus, the proposition follows once we prove that

$$
\bar{\Gamma}\left(n^{\alpha}\right) \longrightarrow 0 \quad P-a . s .
$$

Recall that $E\left[\bar{\Gamma}\left(n^{\alpha}\right)\right]=0$, and by (2.7),

$$
\begin{aligned}
\operatorname{var}\left[\bar{\Gamma}\left(n^{\alpha}\right)\right] & =\operatorname{var}\left[\int_{0}^{n^{\alpha}}\left\langle\varrho_{s}, g_{n^{\alpha}}\left(n^{\alpha}-s\right)\right\rangle d s\right]=2 \int_{0}^{n^{\alpha}}\left\langle\lambda,\left(\int_{0}^{s} S_{s-r} g_{n^{\alpha}}(r) d r\right)^{2}\right\rangle d s \\
& \leq 2 \int_{0}^{n^{\alpha}}\left\langle\lambda,\left[\int_{0}^{s} S_{s-r}\left(\int_{0}^{r} S_{r-l}\left(S_{l} f_{n^{\alpha}}\right)^{2} d l\right) d r\right]^{2}\right\rangle d s \\
& \leq A_{\alpha} \cdot n^{-2 \alpha} \int_{0}^{n^{\alpha}}\left\langle\lambda,\left[s S_{s} f \cdot \int_{0}^{\infty}\left(1 \wedge l^{-d / 2}\right) d l\right]^{2}\right\rangle d s \\
& \leq A_{\alpha} \cdot n^{-2 \alpha} \int_{0}^{n^{\alpha}} s^{2}\left(1 \wedge s^{-d / 2}\right) d s \\
& \leq A_{\alpha} \cdot n^{-\alpha} .
\end{aligned}
$$

Thus for any $\varepsilon>0$,

$$
\sum_{n=1}^{\infty} P\left[\left|\bar{\Gamma}\left(n^{\alpha}\right)\right|>\varepsilon\right] \leq A_{\alpha} \sum_{n=1}^{\infty} \varepsilon^{-2} n^{-\alpha}<\infty
$$

and (2.16) follows by the Borel-Cantelli Lemma.

Acknowledgment This work was done during a visit of Wenming Hong to the University of Minnesota. He would like to thank the Department of Mathematics, University of Minnesota for its hospitality during this visit.

\section{References}

[D93] Dawson, D.A., Measure-valued Markov processes, In: Lect. Notes. Math. 1541, 1-260 (1993), Springer-Verlag.

[DGL02] Dawson, D.A., Gorostiza, L.G., Li, Z.H., Non-local branching superprocesses and some related models, Acta Applicandae Mathematicae, 74 (2002), 93-112.

[H02] Hong, W.M., Longtime behavior for the occupation time of super-Brownian motion with random immigration, Stochastic Process. Appl. 102 (2002), 43-62.

[H03] Hong, W.M., Large deviations for the super-Brownian motion with super-Brownian immigration, Journal of Theoretical Probability, 16 (2003), 899-922.

[H05] Hong, W.M., Quenched mean limit theorems for the super-Brownian motion with super-Brownian immigration, Infinite Dimensional Analysis, Quantum Probability and Related Topics, 8 (2005), 383-396. 
[HL99] Hong, W.M. and Li, Z.H., A central limit theorem for the super-Brownian motion with super-Brownian immigration, J. Appl. Probab. 36 (1999), 1218-1224.

[I86] Iscoe, I., A weighted occupation time for a class of measure-valued critical branching Brownian motion, Probab. Th. Rel. Fields 71 (1986), 85-116.

[IL93] Iscoe, I., Lee, T.Y., Large deviations for occupation times of measure-valued branching Brownian motions, Stoch. Stoch. Rep. 45 (1993), 177-209.

[L93] Lee, T.Y., Some limit theorems for super-Brownian motion and semilinear differential equations. Ann. Probab. 21 (1993), 979-995.

[LR95] Lee, T.Y. and Remillard, B, Large deviation for three dimensional super-Brownian motion. Ann. Probab. 23 (1995), 1755-1771.

[P02] Perkins, E. A., Dawson-Watanabe superprocesses and measure-valued diffusions, Lecture Notes Math. 1781 (2002), 132-318, Springer-Verlag.

[RS05] Rassoul-Agha, F., Seppäläinen, T.: An almost sure invariance principle for random walks in a space-time random environment, Probab. Theory Related Fields 133 , pp. 299-314 (2005).

[Ze04] Zeitouni, O., Random walks in random environment, Lecture notes in Math. 1837 (2004), 193-312, Springer.

[Zh05] Zhang, M., Functional central limit theorems for the super-Brownian motion with super-Brownian immigration, Journal of Theoretical Probability, 18 (2005), 665-685. 